\title{
Qualidade de vida e sintomas de transtornos alimentares em bailarinos
}

http://dx.doi.org/10.11606/1807-5509201800030371

\author{
Nycolle Martins REIS* \\ Andreia PELEGRINI* \\ Leonessa BOING* \\ Adriana Coutinho de Azevedo GUIMARÃES* \\ *Centro de Ciências \\ da Saúde e do \\ Esporte, Universidade \\ Estadual de \\ Santa Catarina, \\ Florianópolis, SC, \\ Brasil.
}

\section{Resumo}

Analisar a relação entre os domínios da qualidade de vida e os sintomas de transtornos alimentares. Participaram 156 bailarinos do 31 $1^{\circ}$ Festival de Dança de Joinville - Santa Catarina, os quais responderam a um questionário autoaplicável contendo as variáveis sóciodemográficas, medidas antropométricas, qualidade de vida (WHOQOL-bref.), Teste de Atitudes Alimentares (EAT-26) e Bulimic Inventory Test Edinburgh (BITE). Observou-se associação significativa entre presença e ausência dos sintomas de anorexia em relação ao sexo feminino e masculino $(p=0,001)$ e as três modalidades de dança $(p=0,018)$, bem como na presença e ausência da escala sintomática de bulimia $(p=0,044)$ na associação com o sexo feminino e masculino. Foram encontrados relações entre o domínio físico da qualidade de vida e os sintomas de anorexia $(p=0,012)$ e de bulimia na escala de gravidade $(p=0,015)$. Em relação à escala sintomática de bulimia percebe-se relação com os domínios físico $(p=0,000)$, psicológico $(p=0,032)$ e social $(p=0,010)$ da qualidade de vida. Os bailarinos do sexo feminino apresentam tendência para a presença de sintomas de anorexia e sintomas de bulimia, bem como as modalidade de ballet clássico e dança contemporânea podem ser fator influenciador. Encontrou-se forte associação entre a presença de sintomas de transtornos alimentares aos domínios da qualidade de vida.

Palavras-chave: Estilo de vida; Anorexia nervosa; Bulimia nervosa; Dança.

\section{Introdução}

O treinamento em dança envolve potencialmente experiências de estresse ${ }^{1}$, decorrente das elevadas demandas físicas e estéticas da modalidade ${ }^{2}$. Dentre tais exigências, encontram-se a busca pela magreza $^{3}$, as experiências como exposição do corpo em público e em frente ao espelho ${ }^{4}$, as observaçôes e comparaçóes do corpo no grupo e o alto nível de competividade ${ }^{1}$. Ainda, quando relacionados a um calendário irregular, incluindo longos dias de treinamentos, ensaios e apresentaçóes, dificultam o ritmo diário normal com padróes biológicos saudáveis ${ }^{5}$, apresentando-se como fator de risco para a saúde e qualidade de vida do bailarino.

Os problemas relacionados à saúde desses indivíduos requerem atenção especial, primeiramente porque a maioria dos bailarinos inicia a carreira cedo ${ }^{2}$, próximo à adolescência, no qual encontram-se mais suscetíveis aos sintomas de transtornos alimentares e emocionais, pois atrelam o sucesso pessoal à aparência física ${ }^{6}$, considerando-se fator de risco ${ }^{1}$. Evidências apontam que a prevalência de sintomas transtornos alimentares é maior entre indivíduos com idade de 12 a 25 anos $^{7}$ principalmente em mulheres ${ }^{6}$ sendo mais frequente neste período a prática de atividades ligadas a forma física ${ }^{7}$. Esses sintomas de transtornos alimentares e emocionais promovem algumas consequências futuras (musculoesqueléticas, metabólicas e os distúrbios alimentares) na saúde e qualidade de vida destes bailarinos ${ }^{2}$.

É importante destacar que a prevalência de sintomas de transtornos alimentares parece ser mais aparente em grupos específicos da sociedade como modelos, atletas e bailarinos ${ }^{8-11}$. Nota-se que há uma forte associação com os distúrbios e fatores específicos como baixa autoestima, padróes pessoais elevados ${ }^{8}$, perfeccionismo ${ }^{4}$, busca pela magreza ${ }^{10} \mathrm{e}$ ambientes 
competitivos $^{3}$. Estes também são observados na ginástica e na dança ${ }^{12}$, mas especialmente no ballet $^{13}$ no qual a busca pela magreza está em ênfase ${ }^{3}$.

São encontradas na literatura, lacunas relacionadas a dança e a qualidade de vida ${ }^{14,15}$, e há ainda menos estudos quando o assunto inclui os sintomas de transtornos alimentares. Estudos que relacionem tais variáveis, identificando a presença ou náo de sintomas de transtornos alimentares nos bailarinos, tem o papel de influenciar positivamente a relação do professor/técnico com o bailarino, a fim de aprimorar técnica e melhorar o desempenho sem prejudicar a saúde destes indivíduos, bem como possibilitar o apoio adequado para os mesmos. Neste sentindo, o presente estudo teve como objetivo analisar a relação entre os domínios da qualidade de vida e os sintomas de transtornos alimentares em bailarinos.

\section{Método}

Trata-se de um estudo transversal composto de 156 bailarinos de ambos os sexos das modalidades Balé Clássico $(n=48)$, Dança Contemporânea $(n=23)$ e Danças Urbanas ( $\mathrm{n}=86$ ), inscritos na categoria avançada (nascidos no ano de 1996, acima de 16 anos), subgênero conjunto, do $31^{\circ}$ Festival de Dança de Joinville na cidade de Joinville/SC, os quais foram selecionados por conveniência.

Foram considerados elegíveis os bailarinos que se propuseram a participar voluntariamente do estudo e que assinaram o termo de consentimento livre e esclarecido. O projeto foi aprovado pelo Comitê de Ética em Pesquisa com Seres Humanos (CEPSH) da Universidade do Estado de Santa Catarina (UDESC) (parecer $n^{\circ}$ 200.140). Os grupos de dança foram contatados pelos pesquisadores por e-mail, telefone, sites de relacionamentos, após divulgada a seleção na mídia pelo festival, para saber do interesse em participar da pesquisa, a fim de fazer um levantamento do número de bailarinos por grupo e estratégia de coleta de dados. Desta forma foi encaminhado por email aos pais e/ou responsáveis de bailarinos menores de 18 anos o termo de consentimento livre e esclarecido (TCLE), sendo entregues posteriormente assinados pelos pais e/ou responsáveis no dia da coleta.

Apesar do regulamento do Festival solicitar idade superior a 16 anos, foram encontrados $(n=11)$ bailarinos com 15 anos, sendo excluídos do presente estudo. Excluiu-se também um bailarino com 57 anos, por diferir-se demasiadamente da idade dos demais participantes do estudo.

A coleta de dados foi realizada durante o mês de julho de 2013, após a aprovaçáo do CEPSH e da organizaçáo do $31^{\circ}$ Festival de Dança de Joinville SC. Os participantes foram esclarecidos sobre o correto preenchimento do questionário e elucidados sobre o objetivo do mesmo. O questionário foi aplicado antes das apresentaçóes da mostra competitiva das respectivas modalidades de dança e respondido de forma presencial com o auxílio das pesquisadoras, sendo respondidos individualmente, com garantia de sigilo das respostas e da identidade dos bailarinos.

Para a coleta de dados foi utilizado um questionário autoaplicável construído por meio de instrumentos validados, dividido em três blocos:

Primeiro bloco: informaçóes gerais contidas de forma objetiva no questionário - idade (em anos completos), sexo (feminino, masculino), modalidade (ballet clássico, dança contemporânea, danças urbanas), escolaridade (ensino fundamental, ensino médio, ensino superior), estado conjugal (com ou sem companheiro), nível econômico (alto, médio, baixo) e variáveis antropométricas (massa corporal e estatura) para cálculo do Índice de Massa Corporal (IMC).

A massa corporal e a estatura foram coletadas por meio de medidas autorreferidas pelos bailarinos. Por tratar-se de adultos em sua maioria, a correspondência entre medidas referidas e aferidas é satisfatória, de modo que o uso deste método tem sido considerado uma alternativa viável em situaçóes em que não é possível a obtenção direta dessas informaçôes ${ }^{16}$, ou quando é preciso tornar o estudo mais rápido $\mathrm{e}$ acessível quanto aos custos ${ }^{17}$. A partir das medidas de massa corporal e estatura calculou-se o IMC dos bailarinos, dividindo estes em três subgrupos: peso normal, baixo peso e excesso de peso.

O nível econômico foi avaliado por meio do instrumento denominado Critério de Classificação Econômica Brasil (CCEB) proposto pela Associação Brasileira de Empresas de Pesquisa ${ }^{18}$, que classifica os indivíduos em estratos (A1, A2, B1, B2, C1, C2, D, E), a partir da soma dos pontos de cada questão. Para fins estatísticos as variáveis foram agrupadas em: nível econômico baixo $(\mathrm{C} 1+\mathrm{C} 2+\mathrm{D}+\mathrm{E})$; médio $(\mathrm{B} 1+\mathrm{B} 2)$ e alto $(\mathrm{A} 1+\mathrm{A} 2)$. 
Segundo bloco: qualidade de vida - avaliada por meio do questionário abreviado World Health Organization Quality of Life (WHOQOL-Bref.), adaptado para Português do Brasil ${ }^{19}$. Trata-se de um questionário composto por 26 questóes (das 100 originais), que se referem às duas últimas semanas. Destas, 24 questóes que abrangem o domínio físico (dor física, energia, locomoção, atividades da vida diária, tratamento médico, trabalho), psicológico (sentimentos positivos, concentração, autoestima, autoimagem, sentimentos negativos, espiritualidade), social (relaçóes pessoais, suporte social, atividade sexual) e meio ambiente (segurança física, habitação, recursos financeiros, serviço de saúde, informaçóes, lazer, ambiente físico e transporte,) e as duas questóes restantes abordam qualidade de vida geral.

Terceiro bloco: sintomas de transtornos alimentares - para a avaliaçáo dos sintomas de transtornos alimentares foram utilizados dois instrumentos. O Teste de Atitudes Alimentares (EAT-26), o qual é, especialmente, utilizado para avaliar os sintomas de anorexia nervosa e já foi traduzido e validado para a população brasileira ${ }^{20}$. O questionário é composto por 26 questóes agrupadas em diferentes aspectos de comportamento alimentar (escala de dieta, escala de bulimia, preocupaçóes com os alimentos e escala de controle oral). As questóes do EAT-26 apresentam seis opções de resposta em escala ordinal (nunca, quase nunca, poucas vezes, às vezes, muitas vezes e sempre) que pontuam de zero a três. Desta forma, as respostas nunca, quase nunca e poucas vezes somam zero ponto, e as respostas às vezes, muitas vezes e sempre pontuam 1,2 e 3 pontos, respectivamente. Na questão 25, a pontuação é conferida de forma inversa às demais, somando-se zero ponto para as alternativas às vezes, muitas vezes e sempre, e 1, 2 e 3 pontos para as alternativas poucas vezes, quase nunca e nunca, respectivamente. A pontuação final do questionário pode variar de 0 a 78 pontos e o ponto de corte estabelecido pelos autores da escala original é $21^{21}$, sendo que indivíduos que somarem 21 pontos ou mais apresentam comportamento alimentar de risco ao desenvolvimento de transtornos alimentares. Assim, o resultado do EAT-26 foi classificado em duas categorias: presença de sintomas (EAT-26 $\geq 21)$ e ausência de sintomas (EAT-26 $<21)^{22}$.

O segundo instrumento utilizado foi o Bulimic Inventory Test Edinburgh (BITE), o qual tem sido utilizado para detectar e avaliar a bulimia nervosa. Este instrumento foi traduzido para o português por Cordás e HochGraF ${ }^{23}$. O questionário é composto por 33 questóes e duas escalas ${ }^{21}$. A primeira escala "sintomática" possibilita uma classificação do grau dos sintomas bulímicos presentes, com no máximo trinta pontos: "compulsão e grande probabilidade de diagnóstico de bulimia" (pontuação $\geq 20$ pontos), "grupo subclínico" (15 a 19 pontos), "padrão alimentar incomum" (10 a 14 pontos) e "ausência de comportamentos típicos da bulimia" ( 0 a 9 pontos). A segunda escala, de "gravidade", fornece um índice baseado na frequência dos comportamentos compulsivos e purgativos. Os resultados podem ser "sem significância (total $\leq 4$ pontos), "clinicamente significante" (5 a 9 pontos) ou "indícios de alta gravidade" ( $\geq 10$ pontos) ${ }^{22}$. Para fins estatísticos os participantes foram classificados na variável BITE sintomas com presença (compulsão e grande probabilidade de diagnóstico de bulimia + grupo subclínico + padrão alimentar incomum) e ausência (ausência de comportamentos típicos da bulimia) de bulimia; e na variável BITE gravidade, com presença (clinicamente significante + indícios de gravidade) e ausência (sem significância) de bulimia.

A análise estatística foi realizada por meio do pacote estatístico Statistical Package for Social Sciences (SPSS) - versão 20.0. Fez-se uso da estatística descritiva, mediante o cálculo de média, desvio padrão e distribuição de frequências. A normalidade dos dados foi verificada por meio do teste Kolmogorov Smirnov. Para a associação das características gerais da amostra com os sintomas de transtornos alimentares (EAT, BITE-gravidade e BITE-sintomas) utilizouse os testes Qui-Quadrado e Exato de Fisher. Já para comparação dos domínios da qualidade de vida com os sintomas de transtornos alimentares utilizou-se o teste U-Mann Whitney (distribuição não normal). O nível de significância estatística adotado foi de $5 \%$.

\section{Resultados}

Observa-se na TABELA 1 associação entre sintomas de anorexia nervosa, sexo e modalidade de dança.
Os resultados apontam maior proporção de sintomas de anorexia nervosa no sexo feminino e nos bailarinos 
Legenda: D.

Contemp $=$ Dança

Contemporânea,

Pres. $=$ presença

de sintomas,

Aus. = ausência

de sintomas,

EAT $=$ Teste de

Atitudes Alimentares,

BITE= Bulimic

Inventory Test

Edinburgh,

GRAV= escala

de gravidade,

SINT= escala

sintomática;

IMC = Índice de

Massa Corporal.

*Qui-Quadrado;

**Exato de Fisher. de dança contemporânea e ballet clássico. Além disso, observa-se associação da escala sintomática de bulimia com o sexo, a qual revela maior proporção de sintomas de bulimia no sexo feminino.

TABELA 1 - Associação dos sintomas de anorexia e bulimia nervosas com variáveis sociodemográficas e status do peso em bailarinos. Joinville, Santa Catarina, 2013.

\begin{tabular}{|c|c|c|c|c|c|c|c|c|c|}
\hline & EAT & & & BITE_GR & & & BITE_SI & & \\
\hline & Pres. & Aus. & $\begin{array}{c}\text { p- } \\
\text { valor }\end{array}$ & Pres. & Aus. & $\begin{array}{c}\text { p- } \\
\text { valor }\end{array}$ & Pres. & Aus. & $\begin{array}{c}\text { p- } \\
\text { valor }\end{array}$ \\
\hline & n(\%) & n(\%) & & n(\%) & $\mathbf{n}(\%)$ & & n(\%) & n(\%) & \\
\hline Sexo* & & & 0,001 & & & 0,256 & & & 0,044 \\
\hline Feminino & $28(31,1)$ & $62(68,9)$ & & $32(43,2)$ & $42(56,8)$ & & $32(43,2)$ & $42(56,8)$ & \\
\hline Masculino & $5(7,8)$ & $59(92,2)$ & & $18(33,3)$ & $36(66,7)$ & & $14(35,9)$ & $40(74,1)$ & \\
\hline Modalidade* & & & 0,01 & & & 0,592 & & & 0,322 \\
\hline Ballet Clássico & $14(29,8)$ & $33(70,2)$ & & $18(46,2)$ & $21(53,8)$ & & $15(38,5)$ & $24(61,5)$ & \\
\hline D. Contemp. & $8(34,8)$ & $15(65,2)$ & & $8(38,1)$ & $13(61,9)$ & & $10(47,6)$ & $11(52,4)$ & \\
\hline $\begin{array}{l}\text { Danças } \\
\text { Urbanas }\end{array}$ & $11(12,9)$ & $74(87,1)$ & & $25(36,2)$ & $44(63,8)$ & & $21(30,4)$ & $48(69,6)$ & \\
\hline Escolaridade* & & & 0,145 & & & 0,297 & & & 0,196 \\
\hline $\begin{array}{l}\text { Ensino } \\
\text { Fundamental }\end{array}$ & $1(100)$ & $0(0,0)$ & & $1(100)$ & $0(0,0)$ & & $1(100)$ & $0(0,0)$ & \\
\hline Ensino Médio & $17(19,8)$ & $69(80,2)$ & & $29(43,3)$ & $38(56,7)$ & & $27(40,3)$ & $40(59,7)$ & \\
\hline $\begin{array}{l}\text { Ensino } \\
\text { Superior }\end{array}$ & $14(20,9)$ & $53(79,1)$ & & $21(35,0)$ & $39(65,0)$ & & $18(30,0)$ & $42(70,0)$ & \\
\hline $\begin{array}{l}\text { Estado } \\
\text { conjugal* }\end{array}$ & & & 0,719 & & & 0,508 & & & 0,202 \\
\hline $\begin{array}{l}\text { Com } \\
\text { companheiro }\end{array}$ & $9(23,1)$ & $30(76,9)$ & & $15(44,1)$ & $19(55,9)$ & & $9(26,5)$ & $25(73,5)$ & \\
\hline $\begin{array}{l}\text { Sem } \\
\text { companheiro }\end{array}$ & $23(20,4)$ & $90(79,6)$ & & $35(37,6)$ & $58(62,4)$ & & $36(38,7)$ & $57(61,3)$ & \\
\hline $\mathrm{IMC}^{* *}$ & & & 0,133 & & & 0,958 & & & 0,393 \\
\hline Peso normal & $30(23,4)$ & $98(76,6)$ & & $43(40,2)$ & $64(59,8)$ & & $39(36,4)$ & $68(63,6)$ & \\
\hline Baixo peso & $1(8,3)$ & $11(91,7)$ & & $4(36,4)$ & $7(63,6)$ & & $5(45,5)$ & $6(54,5)$ & \\
\hline $\begin{array}{l}\text { Excesso de } \\
\text { peso }\end{array}$ & $0(0,0)$ & $9(100)$ & & $3(42,9)$ & $4(57,1)$ & & $1(14,3)$ & $6(85,7)$ & \\
\hline $\begin{array}{l}\text { Nível } \\
\text { econômico* }\end{array}$ & & & 0,232 & & & 0,441 & & & 0,136 \\
\hline Alto & $12(29,3)$ & $29(70,7)$ & & $16(47,1)$ & $18(52,9)$ & & $17(50,0)$ & $17(50,0)$ & \\
\hline Médio & $14(19,7)$ & $57(80,3)$ & & $19(33,9)$ & $37(66,1)$ & & $17(30,4)$ & $39(69,6)$ & \\
\hline Baixo & $6(14,3)$ & $36(85,7)$ & & $16(42,1)$ & $22(55,9)$ & & $12(31,6)$ & $26(68,4)$ & \\
\hline
\end{tabular}

$\mathrm{Na}$ TABELA 2, diferenças significativas foram encontradas entre o domínio psicológico da qualidade de vida e EAT. Ademais, diferenças significativas foram observadas entre o domínio psicológico e a escala gravidade da bulimia nervosa, assim como foram verificadas diferenças entre os domínios físico, psicológico e social e a escala de bulimia sintomática.
Estes resultados apontam que os bailarinos com bulimia nervosa escala gravidade (BITE_GRAV) apresentam maiores prejuízos no domínio psicológico, enquanto os bailarinos com bulimia sintomática (BITE_SINT) apresentam maiores prejuízos nos domínios físico, psicológico e social quando comparados aos bailarinos sem bulimia nervosa $(\mathrm{p}<0,05)$. 
Tabela 2 - Comparação dos domínios da qualidade de vida com a presença e ausência de anorexia e bulimia nervosa em bailarinos. Joinville, Santa Catarina, 2013.

\begin{tabular}{|c|c|c|c|c|c|c|c|c|c|}
\hline \multirow{3}{*}{ 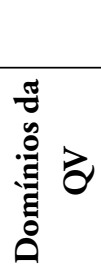 } & \multicolumn{3}{|c|}{ EAT } & \multicolumn{3}{|c|}{ BITE_GRAV } & \multicolumn{3}{|c|}{ BITE_SINT } \\
\hline & Pres. & Aus. & $\mathbf{p}^{*}$ & Pres. & Aus. & $\mathbf{p}^{*}$ & Pres. & Aus. & $\mathbf{p}^{*}$ \\
\hline & $\bar{x}(d p)$ & $\bar{x}(d p)$ & & $\bar{x}(d p)$ & $\bar{x}(d p)$ & & $\bar{x}(d p)$ & $\bar{x}(d p)$ & \\
\hline Físco & $54,6(11,6)$ & $56,8(10,4)$ & 0,338 & $54,6(11,2)$ & $57,6(9,5)$ & 0,158 & $52,6(10,2)$ & $58,5(9,7)$ & $<0,001$ \\
\hline Psicol. & $61,3(10,4)$ & $66,8(10,5)$ & 0,012 & $62,9(10,5)$ & $67,9(10,1)$ & 0,015 & $63,2(10,2)$ & $67,4(10,4)$ & 0,032 \\
\hline Social & $69,9(19,2)$ & $72,4(17,6)$ & 0,461 & $69,5(17,8)$ & $74,3(16,8)$ & 0,115 & $67,5(17,9)$ & $75,2(16,4)$ & 0,010 \\
\hline Amb. & $64,8(19,7)$ & $64,6(14,6)$ & 0,616 & $63,2(18,0)$ & $64,9(15,2)$ & 0,839 & $64,6(17,2)$ & $64,0(15,9)$ & 0,700 \\
\hline
\end{tabular}

QV = qualidade de vida; Psicol. = Domínio Psicológico, Amb. = Domínio Ambiental, Pres. = presença de sintomas, Aus.= ausência de sintomas, EAT $=$ Teste de Atitudes Alimentares, BITE= Bulimic Inventory Test Edinburgh, GRAV= escala de gravidade, SINT= escala sintomática; $p=p$-valor. *U-Mann Whitney.

\section{Discussão}

Observa-se no presente estudo, tendência para o sexo feminino apresentar presença de sintomas de anorexia $(31,1 \%)$ e sintomas de bulimia $(43,2 \%)$. Thomas et al. ${ }^{3}$, comentam que ambientes de dança apresentam-se exigentes em relação às habilidades técnicas e ao peso das bailarinas, principalmente aquelas profissionais. Entendendo que as bailarinas do presente estudo estavam inseridas em ambientes de dança competitivos no Festival de Dança de Joinville acredita-se que essas exigências encontram-se presentes nestas bailarinas. Assim como Penniment e EGan ${ }^{4}$ trazem em seu estudo as bailarinas profissionais, em ambientes competitivos, como mais propensas a desenvolver sintomas de transtornos alimentares. Eles apontam que o elevado perfeccionismo destas, tem grande ênfase na busca pela magreza, na espera por atingir um peso corporal inferior, terão resultados mais positivos nas performances. Ainda, no estudo de Ribeiro e VeigA ${ }^{24}$, as bailarinas profissionais apresentaram maiores estratégias para encontrar o baixo peso quando comparado aos bailarinos.

Em relação às modalidades de dança, notouse que os bailarinos de ballet clássico e dança contemporânea apresentaram presença de sintomas de anorexia na escala EAT, 29,8\% e $34,9 \%$ respectivamente. A literatura aponta maior presença de sintomas de transtornos alimentares em bailarinos provenientes do ballet clássico $^{3,4,9,11}$. Em revisão sistemática atual, cujo objetivo foi verificar a relação dos sintomas de transtornos alimentares e as modalidades de dança, identificou-se que estudos produzidos de 1966 até o ano de 2013, investigaram em sua maioria a modalidade de ballet clássico ${ }^{8}$. Corroborando aos achados de Thomas et al. ${ }^{3}$, que associaram o ambiente do ballet clássico como de maior pressão aos bailarinos quando relacionado à estética, na busca pela magreza. Herbrich et al. ${ }^{25}$ comentam sobre o contexto cultural relacionado ao ballet clássico, a pressão para o corpo magro, a atmosfera competitiva e os treinamentos diários em frente aos espelhos, os quais devem ser considerados na presença de sintomas de transtornos alimentares. Em relação as modalidades de dança, estudiosos como Annus e $\mathrm{S}_{\mathrm{Mith}}{ }^{10}$, acreditam que o que confere risco de desenvolvimento de sintomas de transtornos alimentares nos ambientes de dança são os aprendizados e experiências sobre a necessidade da magreza, como comentários dos professores sobre a importância das dietas e do bailarino magro, a pesagem dos bailarinos nas aulas, modelos de dietas específicos, ou o controle de peso dos outros colegas.

Ao comparar os domínios da qualidade de vida e os sintomas de transtornos alimentares, encontrou-se que os bailarinos com presença de anorexia e bulimia na escala de gravidade e sintomática apresentaram piores escores no domínio psicológico quando comparados aos seus pares sem presença de sintomas. Pode-se relacionar-se o domínio psicológico à percepção do próprio bailarino, nesse sentido, estudos apontam que as pressóes de si mesmo pela busca do corpo perfeito, o "corpo de bailarino" 3,8 , 
associado com a baixa autoestima pode estar vinculado ao desenvolvimento de sintomas de transtornos alimentares ${ }^{26}$. O ambiente de dança, pode ser considerado uma subcultura que amplia as pressóes já existentes na sociedade, a fim de tornar aceitável as expectativas e estereótipos provocados por outros do mesmo meio, depositando bailarinos em risco para o desenvolvimento de sintomas de transtornos ${ }^{6}$. Neste sentido, a expectativa da conquista de uma silhueta magra utilizando a restrição alimentar, pode levar os bailarinos a cair em um confronto psicológico fazendo-os a acreditar que o alcance de um corpo esguio trará ascensão social, melhor aparência física e elevará a autoestima ${ }^{10}$.

Em relação à escala sintomática de bulimia, percebe-se que os bailarinos com presença de sintomas de distúrbio alimentar apresentaram piores escores nos domínios físico, psicológico e social. No domínio físico, pode-se fazer uma reflexão com Anshel ${ }^{6}$ salientando que o ballet clássico tem baixo percentual de perda calórica quando comparado a outros esportes, e junto a grande exigência de perda de peso (exigência pela magreza), neste meio, torna-se quase normal a presença de sintomas de transtornos alimentares. Neste contexto, as altas demandas físicas e exigências máximas dos bailarinos pode levar a uma qualidade de vida ruim $^{2}$. Com elevado número de ensaios e as exigências dos mesmos, altera o sono desses bailarinos, modificando os padróes biológicos normais e alterando o ritmo circadiano, sendo um fator de risco também para o surgimento de lesóes 5 .

No domínio social, associa-se aos feedbacks dos professores, no qual os bailarinos que recebem críticas sobre seu corpo e peso, estáo mais propensos aos sintomas de transtornos alimentares ${ }^{26}$. O estudo de AnNus e AGNes ${ }^{10}$ associou os relatos de aprendizagem sobre a importância da magreza durante as aulas e o ambiente da dança com a presença de sintomas de transtornos alimentares. Nesse sentido, Monteiro e Correa ${ }^{11}$ ressaltam que os professores têm papel considerável no desenvolvimento dos sintomas de transtornos, uma vez que comentários e observaçóes alteram o psicológico do bailarino. As experiências proporcionadas pela dança, como exposição do corpo em público e em frente ao espelho ${ }^{4}$, e as observaçóes e comparações do corpo em grupo ${ }^{1}$ podem ser fatores que influenciam o domínio social em relação aos sintomas de transtornos alimentares.
As limitaçóes deste estudo podem estar associadas à coleta de dados, pelo fato de ocorrerem paralelas as apresentaçóes dos grupos no Festival de Dança de Joinville. Percebe-se como uma grande dificuldade o fato dos bailarinos pausarem suas atividades para responder ao questionário. Por tratar-se, de um Festival com muitas atividades ocorrendo ao mesmo tempo, os grupos estavam dispersos no local, com integrantes em atividades diferentes, cursos, palestras, ensaios de palco, em alojamentos, na feira da sapatilha. Outro fator limitante relacionado ao número de bailarinos por modalidade, está interligado com a ocorrência de que foram incluídos no estudo apenas bailarinos inscritos na modalidade conjunto. No entanto, os trios também foram incluídos na categoria conjuntos, por haverem poucos inscritos e aprovados. Ainda, os conjuntos de danças urbanas possuíam mais integrantes, quando comparados aos conjuntos de dança contemporânea e ballet clássico, pela distinção da modalidade em si. Além disso, as variáveis foram avaliadas por meio de um questionário de autorrelato, o que representa uma limitação para esse tipo de estudo, uma vez que as taxas do diagnóstico podem apresentar distorçôes.

Tendo posto, sugere-se estudos futuros que relacionem os transtornos alimentares e a qualidade de vida em bailarinos em diferentes locais de atuação, além do ambiente competitivo aqui abordado. Neste sentido, um estudo interessante resultaria da análise das variáveis citadas por meio da comparação entre bailarinos competitivos (como os da amostra) e nãocompetitivos (recreacionais ou profissionais). Sugere-se também a exploração das variáveis, já abordadas neste estudo, de forma separada ou em conjunto com as demais variáveis contidas no ambiente da dança.

Conclui-se que bailarinos do sexo feminino podem apresentar uma tendência para a presença de sintomas de anorexia e sintomas de bulimia, bem como as modalidades de ballet clássico e dança contemporânea podem ser fator influenciador. Ainda, encontrou-se diferença nos valores médios da presença de sintomas de transtornos alimentares aos domínios físico, psicológico e social da qualidade de vida. Possibilitando a reflexão para profissionais da área, sobre a influência de aspectos interligados com a prática de dança, como a busca pelo corpo magro, a rotina de ensaios e apresentaçóes, o ambiente competitivo, a imagem corporal distorcida, com a presença de sintomas de transtornos alimentares em bailarinos. 


\title{
Conflito de interesses
}

Não há conflito de interesses entre os autores deste estudo.

\begin{abstract}
Quality of life and eating disorders symptoms in dancers

To analyze the relationship between the domains of quality of life and symptoms of eating disorders. 156 dancers from $31^{\circ}$ Festival de Joinville Dance - Santa Catarina participated in the study, who completed a self-administered questionnaire containing sociodemographic variables, anthropometric measurements, quality of life (WHOOOL-bref.), Eating Attitudes Test (EAT-26) and Bulimic Inventory Test Edinburgh (BITE). A significant association between the presence and absence of symptoms of anorexia compared to female and male dancers $(p=0.001)$ and three modalities of dance $(p=0.018)$ and in the presence and absence of symptomatic scale bulimia ( $p=0.044)$ in association with the female and male. Relations between the quality of life and symptoms of anorexia $(p=0.012)$ and bulimia were found in the scale $(p=0.015)$. Regarding the symptoms of the scale bulimia was perceived a relationship with the physical scale $(p=0.000)$, psychological scale $(p=0.032)$ and social scale $(p=0.010)$ with the quality of life. The female dancers are prone to the presence of symptoms of anorexia and symptoms of bulimia, as well as the modality of classical ballet and contemporary dance can be influential factor. Found a strong association between the presences of symptoms of eating disorders to the domains of quality of life.
\end{abstract}

KeYwords: Life style; Anorexia nervosa; Bulimia nervosa; Dancing.

\section{Referências}

1. Toro J, Guerreiro M, Sentis J, Castro J, Puértolas C. Eating disorders in ballet dancing students: problems and risk factors. Eur Eat Disord Rev. 2009;17(1):40-9.

2. Hincapié CA, Morton EJ, Cassidy JD. Musculoskeletal injuries and pain in dancers: a systematic review. Arch Phys Med Rehabil. 2008;89(9):1819-29.

3. Thomas JJ, Keel PK, Heatherton TF. Disordered eating attitudes and behaviors in ballet students: examination of environmental and individual risk factors. Int J Eat Disord. 2005;38(3):263-8.

4. Penniment KJ, Egan SJ. Perfectionism and learning experiences in dance class as risk factors for eating disorders in dancers. Eur. Eat. Disorders Rev. 2012;20(1):13-22.

5. Fietze I, Strauch J, Holzhausen M, Glos M, Theobald C, Lehnkerin H; Penzel T. Sleep quality in professional ballet dancers. Chronobiol Int. 2009;26(6):1249-62.

6. Anshel MH. Sources of disordered eating patterns between ballet dancers and non-dancers. J Sport Behav. 2004;27(2):115-33.

7. Oliveira DV, Alves KM, Barbosa CP. Prevalência de transtornos alimentares em adolescentes praticantes de ballet clássico. Conexôes. 2013;11(1):171-87.

8. Arcelus J, Witcomb GL, Mitchell A. Prevalence of eating disorders amongst dancers: a systemic review and meta-analysis. Eur Eat Disord Rev. 2014;22(2):92-101.

9. Ringham R, Klump K, Kaye W, Stone D, Libman S, Stowe S, Marcus M. Eating disorder symptomatology among ballet dancers. Int J Eat Disord. 2006;39(6):503-8.

10. Annus A, Smith GT. Learning experiences in dance class predict adult eating disturbance. Eur Eat Disord Rev. 2009; 17(1):50-60.

11. Monteiro MF, Correa MM. Transtornos alimentares em bailarinas clássicas adolescentes. Rev Bras Promoc Saude. 2013;26(3):396-403. 
12. Kravchychyn ACP, Silva DF, Machado FA. Relação entre estado nutricional, adiposidade corporal, percepção de autoimagem corporal e risco para transtornos alimentares em atletas de modalidades coletivas do gênero feminino. Rev Bras Educ Fís Esporte. 2013;27(3):459-66.

13. Wyon MA, Hutchings KM, Wells A, Nevill AM. Body mass index, nutritional knowledge, and eating behaviors in elite student and professional ballet dancers. Clin J Sport Med. 2014;24(5):390-6.

14. Rocha MD, Almeida CM. Dança de salão, instrumento para a vida. Movimento e Percepçáo. 2007;7(10):76-89.

15. Silva TMG, Simas JPN. Qualidade de vida de bailarinos de dança hip hop. Trabalho de Conclusão de Curso; (Graduação em Educação Física) - Universidade do Estado de Santa Catarina; 2010.

16. Barbosa AR, Pelegrini A, Borgescandi LJ, Coqueiro RS, Araújo VC. Medidas auto-referidas são válidas para a avaliação do estado nutricional na população brasileira? Rev Bras Cineantropom Desempenho Hum. 2009;11(1):113-9.

17. Silveira EA, Araújo CL, Gigante DP, Barros AJD, Lima MSD. Validaçáo do peso e altura referidos para o diagnóstico do estado nutricional em uma população de adultos no Sul do Brasil. Cad Saude Publica. 2005;21(1):235-45.

18. Associação brasileira de empresas de pesquisa. Critério de classificação econômica Brasil. São Paulo; 2012.

19. Fleck MPA, Louzada S, Xavier M, Chachamovich E, Vieira G, Santos L, Pinzon V. Aplicação da versão em português do instrumento abreviado de avaliação da qualidade de vida "WHOQOL-bref”. Rev Saúde Pública. 2000;34(2):178-83.

20. Bighetti F. Tradução e validação do Eating Attitudes Test (EAT-26) em adolescentes do sexo feminino na cidade de Ribeirão Preto, SP [dissertação de mestrado]. Ribeirão Preto: Escola de Enfermagem de Ribeirão Preto, Universidade de São Paulo; 2003.

21. Garner DM, Olmsted MP, Bohr Y, Garfinkel PE. The eating attitudes test: psychometric features and clinical correlates. Psychol Med. 1982;12(4):871-8.

22. Belling MTC. A auto-imagem corporal e o comportamento alimentar de adolescentes do sexo feminino em Belo Horizonte [dissertação de mestrado]. Belo Horizonte: Faculdade de Medicina, Universidade Federal de Minas Gerais; 2008.

23. Cordas TA, Hochgraf PB. O "BITE”: instrumento para avaliação da bulimia nervosa: versão para o português. J Bras Psiquiatr. 1993;3(42):141-4.

24. Ribeiro LG, Veiga GV. Risk behaviors for eating disorders in Brazilian dancers. Int J Sports Med. 2010;31(4):283-8.

25. Herbrich L, Pfeiffer E, Lehmkuhl U, Schneider N. Anorexia athletica in pre-professional ballet dancers. J Sports Sci. 2011;29(11):1115-23.

26. Goodwin H, Arcelus J, Marshal S, Wicks S, Meyer C. Critical comments concerning shape and weight: associations with eating psychopathology among full-time dance students. Eat Weight Disord. 2014;19(1):115-8.

\author{
ENDEREÇO \\ Nycolle Martins Reis \\ Rua Tereza Lopes, 734 -Campeche \\ 88066-065 - Florianópolis - \\ Santa Catarina - BRASIL \\ e-mail: nycolle_mr@hotmail.com
}

Recebido para publicação: 12/02/2015

$1^{\text {a }}$. Revisão: 13/01/2017

Aceito: 03/08/2017 